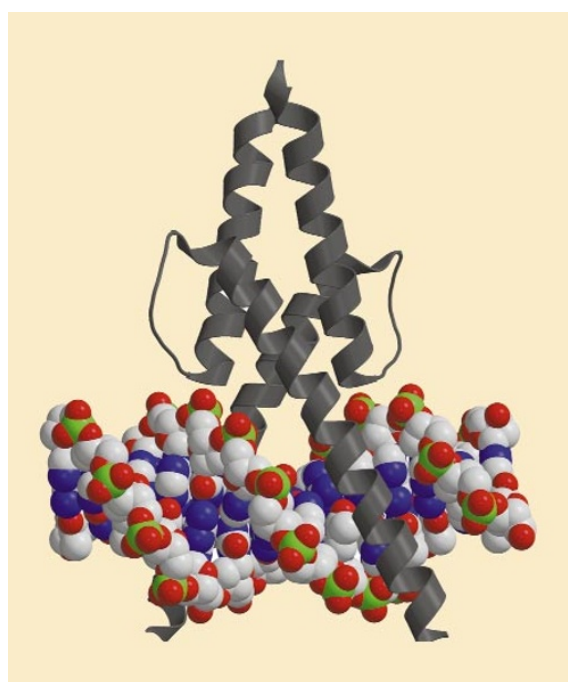

Figure 1 Three-dimensional representation of the structural elements of a basic helix-loop-helix (bHLH) protein (grey). Note that sequence-specific DNA recognition requires the formation of a bHLH protein dimer. Winston and Gottesfeld ${ }^{2}$ show that contacts between residues in the loop regions and base pairs outside the core recognition sequence contribute significantly to energetically favourable DNA-protein interactions. This figure is based on the co-crystal structure of the bHLH protein MyoD bound to its DNA recognition element ${ }^{5}$.

different libraries of Deadpan variants one in which the length of the loop region was modulated, and another in which the composition of the 12 amino acids that make up the full-length loop was systematically varied. Using stepwise solid-phase peptide synthesis, the first bHLH library, containing successive single amino-acid deletions from both the amino- and the carboxy-terminal ends of the loop region, was quickly generated. This approach greatly reduces the drudgery associated with traditional recombinant DNA techniques, such as gene construction, protein expression and purifi-cation, and then characterization of the individual gene products. Twenty-six bHLH loop variants were generated in a few days, the time usually required to produce a single construct by recombinant techniques.

Winston and Gottesfeld took advantage of the power of solid-phase peptide synthesis to produce a second library of bHLH variants containing unnatural peptidyl linkers. Specifically, an alanine- $O$-glycine depsipeptide unit (a peptide that contains an amideto-ester substitution) was systematically scanned through 11 positions in the Deadpan loop. Substitution of the depsipeptide unit allows removal of the side chains of adjacent amino acids (the chemical equivalent of alanine scanning). The positions of the deleted side chain can be readily identified by selective cleavage of the ester linkage followed by characterization of the resultant cleavage products by matrix-assisted laser desorption mass spectrometry.

The DNA-binding activities of both libraries were quickly screened by using a sequence-specific DNA-affinity resin containing the DNA recognition element. Deletion variants of the loop region that retained the ability to bind the resin with normal affinity were characterized in a highthroughput fashion with the mass spectrometry technique. Winston and Gottesfeld found that Deadpan tolerates only a small deletion of the centre of the loop without severely compromising DNA binding.

The identity of amino acids in the loop that might contribute to DNA binding was similarly assessed by DNA selection, followed by selective cleavage coupled with mass spectrometry. The authors show that the replacement of a single lysine residue (lysine 80 in Deadpan) by the depsipeptide unit severely reduces DNA-binding affinity. They further demonstrate that replacement of lysine 80 by unnatural amino acids greatly affects DNA affinity, implicating this loop residue as a central player in DNA binding. In the co-crystal structure of a related bHLH factor, Max, the corresponding lysine (lysine 57) makes contacts with the DNA phosphate backbone, and does so in a region outside the core-DNA recognition element.

This work tells us a great deal about sequence-specific DNA recognition, especially as it pertains to a related bHLH family member, the proto-oncoprotein Myc. The search for Myc target genes has often been based solely on the presence of a sixnucleotide, core Myc-binding site in the promoter region of these target genes. But there are two problems with this strategy. First, the Myc recognition sequence is also recognized by several other members of the bHLH class (including Deadpan); second, Myc binding still occurs in vitro even if the base pairs within the core are changed ${ }^{6}$.

Evidence has been accumulating that nucleotides outside the core DNA sequence might influence binding; indeed, detailed examination of Myc-binding sites in vivo has revealed a predominance of guaninecytosine dinucleotides flanking the core recognition sequence ${ }^{7}$. If, as Winston and Gottesfeld show, interactions between bHLH loop residues and DNA occur outside the core six nucleotides, it seems plausible that the effects of flanking nucleotides might further restrict the number of possible Mycbinding sites within the genome. Moreover, given that the largest variations in sequence between members of the bHLH family occur in the loop regions ${ }^{1}$, the results of Winston and Gottesfeld also pave the way for identifying a molecular basis for how different bHLH proteins discriminate between all possible binding sites.

More generally, the new work shows the power of chemical synthesis methods coupled with high-resolution mass spectrometry. Winston and Gottesfeld screened and

\section{The stellar..........}

This false-colour image of the nearby galaxy IC342 shows the relationship between gas, stars and star formation. The blue features are clusters of stars, whereas the red in the outer region is the atomic hydrogen gas. Green indicates the molecular hydrogen gas, out of which new stars are forming, and yellow regions are where the atomic and molecular gas overlap.

This map of the molecular gas was obtained by Jean Turner of the University of California at Los Angeles, using 'on the fly' mapping at the 12metre telescope of the National Radio Astronomy Observatory at Kitt Peak.

IC342 is a spiral galaxy, like the Milky Way, and the spiral structure is obvious in the distribution of the gas, which changes from

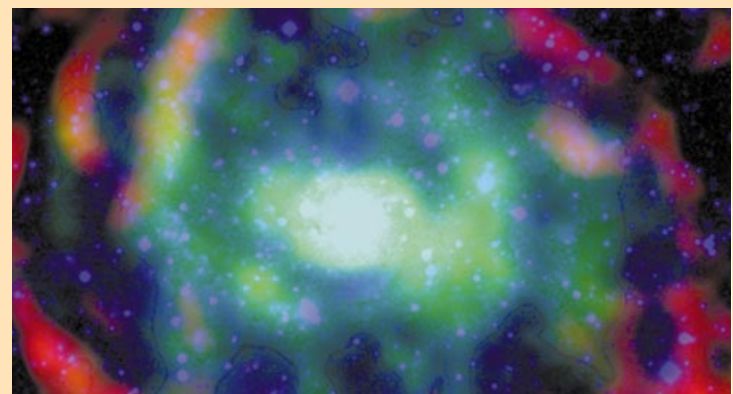

predominantly molecular in the inner region, to predominantly atomic in the outer part. The gas forms a bar-like structure around the nucleus, which is believed to play a role in funnelling gas towards the centre, thereby supplying the fuel to form new stars.

Curiously, the green ring around the nucleus, which indicates a lot of molecular gas, does not seem to be associated with the formation of new stars.

The relationship between the phases of gas and the formation of new stars is still not completely understood. Although it is apparent that in many places simply having lots of gas on hand is sufficient to produce stars, this is not universally true. As we produce higher-resolution maps of the distribution of gas in galaxies, we will eventually sort out the physics of what is really happening.

Leslie Sage 